\title{
Lateral Ankle Ligament Repair: Evolution of Technique from Open to Arthroscopy
}

\author{
${ }^{1}$ Kushal Nag, ${ }^{2}$ Ken Jin Tan
}

\begin{abstract}
One of the most common injuries of the lower extremity is the lateral ankle sprain. In recent years, with greater emphasis on physical fitness, the chronically painful and unstable lateral ankle is an increasing common clinical finding presenting to the outpatient clinic. If residual instability and pain is still present after a course of physiotherapy, the patient should be presented with surgical options to restore ankle stability. Several surgical techniques have been described for the treatment of chronic ankle instability. Recently, some authors have developed arthroscopic suture anchor techniques to repair the lateral ankle ligaments with excellent results. The authors' aim is to give an overview of the various techniques for repair or reconstruction of the lateral ligament complex with special emphasis on the arthroscopic lateral ligament reconstruction.
\end{abstract}

Keywords: Ankle ligament repair, Arthroscopy, Surgical technique.

How to cite this article: Nag K, Tan KJ. Lateral Ankle Ligament Repair: Evolution of Technique from Open to Arthroscopy. J Foot Ankle Surg (Asia-Pacific) 2014;1(2):65-68.

Source of support: Nil

Conflict of interest: None

\section{INTRODUCTION}

One of the most common injuries of the lower extremity is the lateral ankle sprain. In recent years, with greater emphasis on physical fitness, the chronically painful and unstable lateral ankle is an increasing common clinical finding presenting to the outpatient clinic. The mechanism of injury in lateral ankle sprains most commonly involves excessive plantarflexion and inversion of the ankle. The most commonly injured ligament is the anterior talofibular ligament (ATFL), followed by the calcaneofibular ligament (CFL). Patients with acute ankle sprains can be successfully managed with conservative treatment, such as bracing and physical therapy. However, approximately 15 to $20 \%$ of patients will remain symptomatic, with the most commonly described complaints being ankle weakness, giving way, pain and,

\footnotetext{
${ }^{1}$ Consultant, ${ }^{2}$ Assistant Professor

${ }^{1}$ Department of Orthopedics, Apollo Gleneagles, Kolkata West Bengal, India

${ }^{2}$ Department of Orthopedics, National University Hospital Singapore
}

Corresponding Author: Kushal Nag, Consultant, Department of Orthopedics, Apollo Gleneagles, Kolkata, West Bengal India, Phone: 91-9836404945, e-mail:nag.kushal@gmail.com occasionally, stiffness. ${ }^{1}$ If residual instability and pain is still present after a course of physiotherapy, the patient should be presented with surgical options to restore ankle stability. ${ }^{2}$ Historically, lateral ligament repair or reconstruction usually involved open or mini open techniques. Several surgical techniques have been described for the treatment of chronic ankle instability. They can be divided into anatomic repair, non-anatomic reconstruction, and anatomic reconstruction. Recently, some authors have developed arthroscopic suture anchor techniques to repair the lateral ankle ligaments with excellent results. ${ }^{3}$ The authors' aim is to give an overview of the various techniques for repair or reconstruction of the lateral ligament complex with special emphasis on the arthroscopic lateral ligament reconstruction.

\section{ANATOMIC CONSIDERATIONS}

A thorough understanding of the anatomy of the lateral ligament complex is absolutely necessary for a correct ankle ligament repair. The ATFL is a flat, quadrilateral ligament which originates from the anterior margin of the lateral malleolus. From here, it runs anteromedially to the insertion point at the talar body immediately anterior to the joint surface articulating with the lateral malleolus. The ligament is almost horizontal to the ankle in the neutral position but moves upward in dorsiflexion and downward in plantarflexion. This ligament is in close contact with the capsule of the ankle joint, and has a double-band morphology.

The CFL is a thick, cordlike ligament that originates at the anterior edge of the lateral malleolus. The origin of this ligament, however, does not reach the tip of the malleolus, which remains free from ligamentous insertions. In the neutral position, the ligament courses backward, downward, and medially to insert in a small tubercle in the posterior region of the lateral calcaneus posterior to the peroneal tubercle. This ligament becomes horizontal during extension and vertical in flexion, remaining tense throughout its entire arc of motion. The ligament is relaxed in the valgus position and tense in the varus position. ${ }^{5}$

\section{HISTORICAL REVIEW OF THE SURGICAL TECHNIQUES}

Historically, ankle stabilization surgical techniques have primarily been devised to correct either a single 
ligamentous repair involving the ATFL or a double ligamentous repair involving both the ATFL and CFL.

\section{Double Ligamentous Lateral Ankle Repair}

The split peroneus brevis lateral ankle stabilization or the modified evans has proven to be a dependable surgical technique for stabilization of the unstable ankle. In this procedure, we transfer of one half of the peroneus brevis tendon through the fibula, and suture it to the fibular periosteum where it acts as a tenodesis (Fig. 1). Chrisman and Snook used the peroneus brevis tendon as a modification of the procedure by transferring it through a canal in the fibula, reattached it to the lateral aspect of the calcaneus, and then to the peroneus brevis tendon (Fig. 2). Hambly further modified the procedure by splitting the peroneus longus rather than the peroneus brevis. The tendon is passed through the fibula, sutured through the lateral aspect of the talus, and then inserted into the CFL ligament. This is usually accomplished through a single curvilinear hockey stick incision on the lateral aspect of the ankle. Due to the amount of dissection and necessity of securing the transferred tendon graft to the body of the calcaneus and or the talus in a trephine hole, prolonged immobilization and postoperative rehabilitation is necessary which prolongs the patient's return to normal daily activities.

\section{Single Ligamentous Lateral Ankle Repair}

Watson-Jones utilized the entire peroneus brevis tendon, leaving its distal insertion intact. The tendon is passed through a canal in the fibula, into the neck of the talus, back through the fibula, and then attached to itself posteriorly at the level of the fibula. Lee modified the Watson-Jones procedure due to the technical difficulty in obtaining adequate tendon length for transfer. The Lee modification utilizes the entire peroneus brevis tendon, passing it posterior to anterior through a canal in the

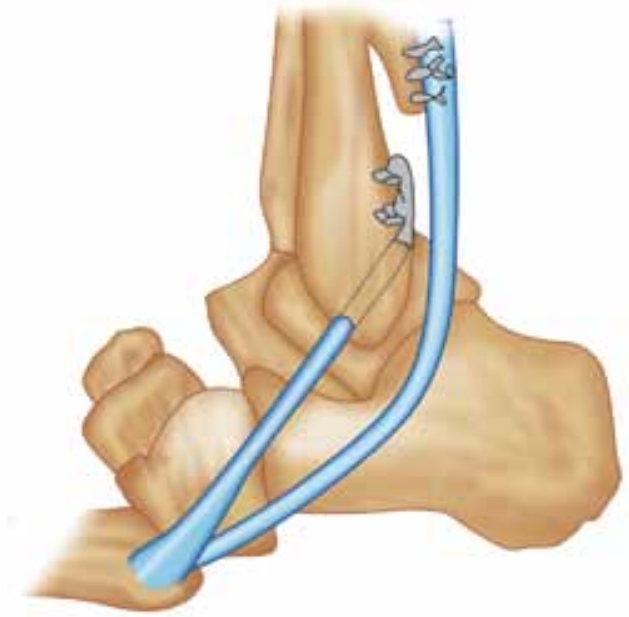

Fig. 1: Evans procedure. In modified Evans, the brevis tendon is split instead of using the tendon as a whole fibula and suturing the peroneus brevis tendon distally and inferiorly upon itself (Fig. 3).

\section{ARTHROSCOPIC LATERAL LIGAMENT RECONSTRUCTION}

\section{Methods}

In a period of over 10 months from April 2013 to January 2014, eight patients (all males with average age of 20 years, range 17 to 26) underwent arthroscopic lateral ligament reconstruction. The right ankle was affected in six cases and the left in two cases. Mean follow-up is 6 months, range 2 to 8 months. All patients had sustained an inversion injury of the ankle during sports activity. Patients reported an instability sensation of the ankle during activity with occasional pain. None of the patients had sustained a foot or ankle fracture. Physical examination showed no foot deformity. Range of motion, anterior drawer test and talar tilt were performed clinically before surgery and compared with the uninjured contralateral side. The anterior drawer test and talar tilt findings were positive in all ankles as compared to the contralateral side. Routine radiographs showed no abnormalities. Preoperative magnetic resonance imaging (MRI) of the ankle was performed in all cases. MRI scan showed chronic injury of the ATFL in all cases, however there was no evidence of osteochondral lesions of the ankles.

\section{Operative Technique}

A suture passer (Microsuture Lasso curved $70^{\circ}$, Arthrex, Naples, FL) and double-loaded Bio-SutureTak Suture Anchor $(3 \times 14.5 \mathrm{~mm}$ Arthrex, Naples, FL) were used in this procedure.

Spinal anesthesia was used. The patient was placed in supine position with the affected extremity rotated internally with toes pointing toward the ceiling with a sandbag under the ipsilateral hip. A thigh tourniquet was used.

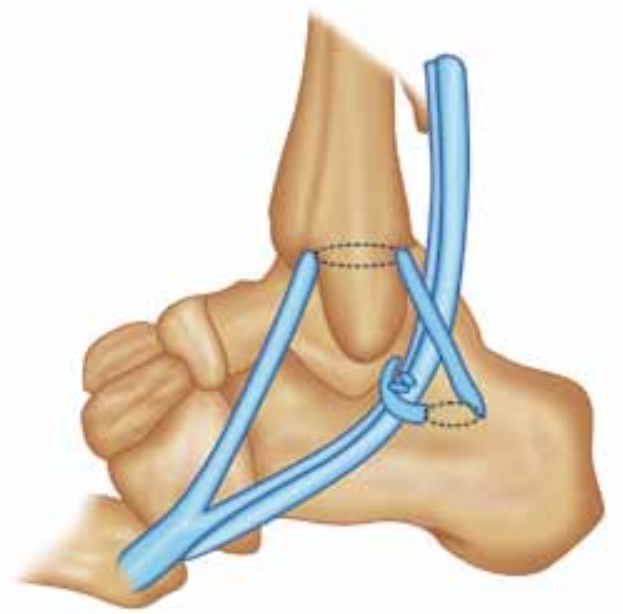

Fig. 2: Chrisman-Snook procedure. Some surgeons prefer splitting the brevis tendon before rerouting it 


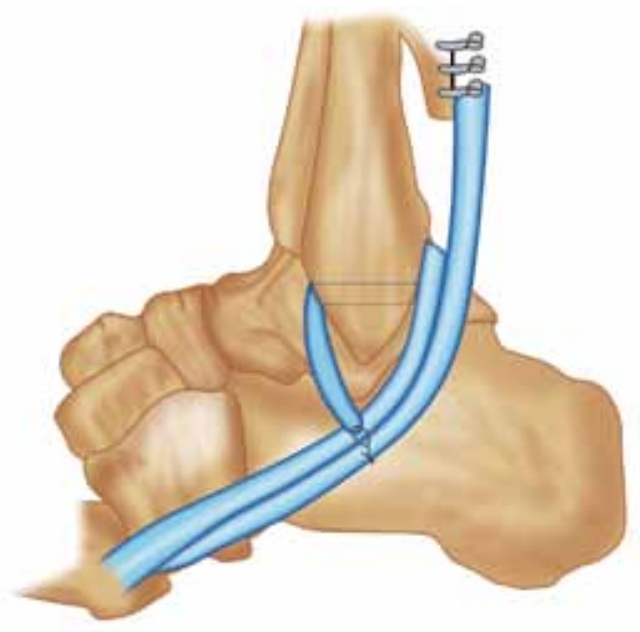

Fig. 3: Lee modification of the Watson-Jones reconstruction of the lateral ligaments using the peroneus brevis

The following landmarks were marked: the anterior joint line, by moving the joint through plantar and dorsiflexion, the tibialis anterior tendon, the peroneus tertius tendon which is present in $90 \%$ of cases (in its absence, the extensor digitorum longus tendon is used as a reference); the lateral malleolus; the superficial peroneal nerve; the peroneal tendons and the extensor retinaculum. The superficial peroneal nerve and its subcutaneous course becomes evident with inversion of the ankle and plantar flexion of the fourth toe. ${ }^{6}$

Distraction of the ankle was not used during the arthroscopic procedure. The anterior capsular insertion into the tibia and talus occurs at a distance from the articular surface. This anatomic feature creates a potential anterior working space when the ankle is in neutral dorsiflexion. ${ }^{7}$ Dorsiflexion of the ankle even without distraction enables good visualization and easy access to the lateral and medial gutter of the ankle. ${ }^{8}$

A standard anterolateral and anteromedial portal was used in this inside out lateral ligament reconstruction technique. Arthroscopically, ATFL injury was defined

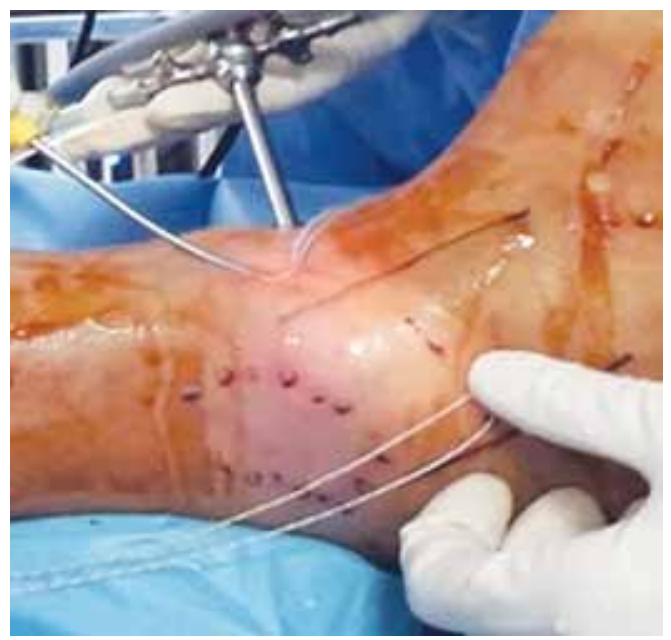

Fig. 5: The Microsuture passer being used to takes bites into the ATFL remnant and being passed out through the skin

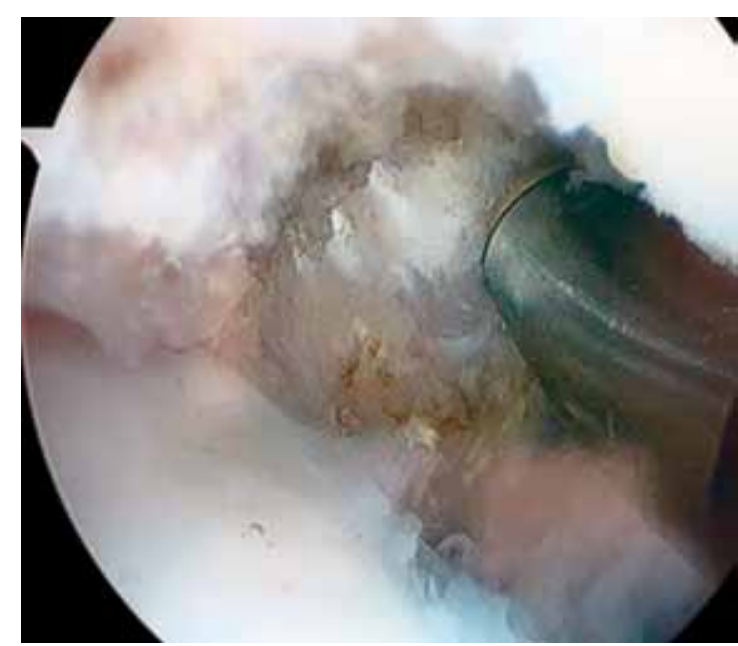

Fig. 4: The radiofrequency probe to clear the footprint of the ATFL at the anteroinferior border of the fibula

as complete disinsertion of the fibular attachment with vision of the ATFL footprint. The footprint for the fibular attachment of the lateral collateral ligaments was debrided with a shaver introduced through the anterolateral portal (Fig. 4). The suture anchor was then drilled into the footprint of the torn ATFL through the anterolateral portal, care was taken not to violate the fibular articular cartilage. The suture passer was then introduced through the anterolateral portal, and under direct arthroscopic visualization, the ligament was penetrated from lateral to medial and brought out through zone marked on the skin for the extensor retinaculum (Fig. 5). This step was repeated till all the four color coded fiber wire threads were brought out through the skin. A small incision was made on the skin near the puncture holes for the threads and with the help of an arthroscopic probe, the four threads were delivered through the incision. Then with the ankle held in eversion and neutral dorsiflexion the color coded threads were tied to each other (Fig. 6). This step completed the repair of the torn ATFL with reinforcement by the extensor retinaculum.

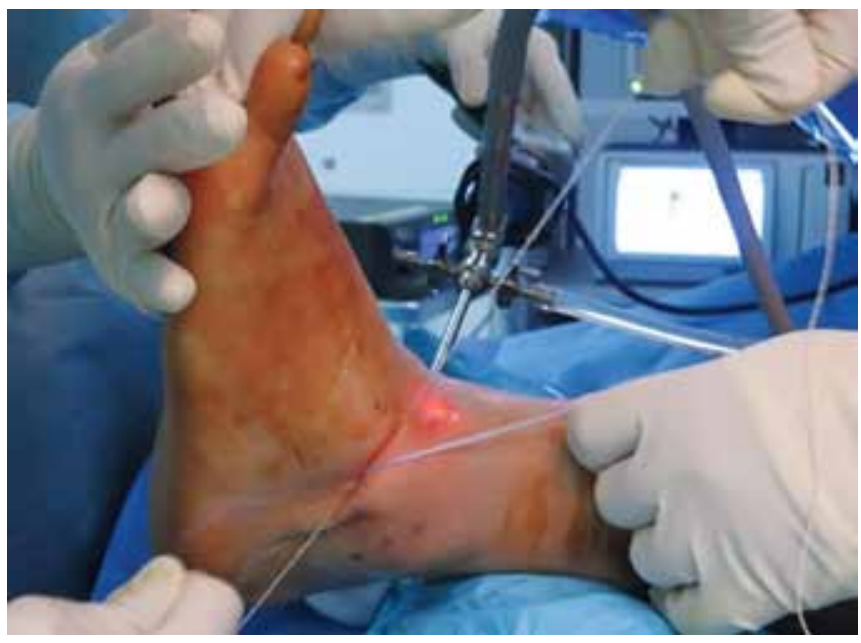

Fig. 6: The foot held in eversion and neutral dorsiflexion, the color coded fiberwire threads are tied to each other to complete the reconstruction 
A posterior plaster splint with the ankle in neutral position and eversion were applied and maintained for 14 days. The splint was then removed at 14 days and was switched to a removable walking boot with eversion wedge to begin partial weight bearing with an assistive device for the next 2 weeks. Full weightbearing was allowed at 4 weeks with intermittent ankle mobilization. At 6 weeks postoperative, the boot was removed and ankle strengthening exercises started gradually. Patient was allowed full weightbearing with a soft ankle guard. Patients were allowed return to noncontact sports at 3 months post-surgery.

\section{RESULTS}

Eight patients underwent the inside out arthroscopic lateral ligament reconstruction. At follow-up, all patients reported subjective improvement in their ankle stability. Objective assessment with the AOFAS score is being done at follow-up. However, we do not have sufficient followup at present to arrive at a conclusion regarding the efficacy of this procedure.

\section{DISCUSSION}

The arthroscopic reconstruction of ankle ligaments continues to evolve. Due to several intra-articular conditions associated with ankle instability that can contribute to pain and dysfunction, arthroscopic evaluation may be beneficial. ${ }^{9}$ Chondral lesions, loose bodies, or other intra-articular pathology have been found in 66 to $93 \%$ of unstable ankles on arthroscopic examination. ${ }^{10} \mathrm{~A}$ consensus is evolving regarding an increasing role for arthroscopy as a definitive treatment. ${ }^{11}$ Hawkins was the first to describe an arthroscopic approach to lateral collateral ankle ligament repair with a stapling technique. ${ }^{12}$ A suture anchor technique was later developed to avoid problems caused by the prominent staples. ${ }^{13}$ More recently, other authors have developed arthroscopic assisted suture anchor techniques to repair lateral collateral ankle ligament instability with excellent result. ${ }^{11}$ Superficial peroneal or sural nerve neuritis and discomfort due to a prominent anchor or suture knot have been the most frequent complications reported..$^{14}$ Arthroscopic experience of the surgeon and a thorough knowledge of foot and ankle anatomy are critical to reduce the risk of complications during the procedure. Caution on the part of the surgeon is warranted when creating portals, introducing or using instruments, to reduce the risk of nerve injuries. The superficial peroneal nerve should be marked to avoid its injury when creating the anterolateral portal. The use of a cannula in the anterolateral portal might help to avoid peroneal nerve injuries during the procedure. Previous studies indicate that open surgery or arthroscopic assisted procedures produce similar satisfactory results. Arthroscopy has advantages over open surgery of being a less aggressive surgery. Morbidity can be lower and the cosmetic result is better than open surgery. More importantly, concomitant intra-articular injuries can be evaluated and treated arthroscopically before the ligament repair. Anterolateral soft tissue impingement can also be dealt with at the same setting through the same portals. It can also be easily converted to an open technique, if required.

\section{REFERENCES}

1. DiGiovanni BF, Partal G, Baumhauer JF. Acute ankle injury and chronic lateral instability in the athlete. Clin Sports Med 2004;23(1):1-19.

2. Hua Y. Modified broström procedure plus ankle arthroscopy may be effective for ankle instability. Arthroscopy 2010;26(4): 524-528.

3. Acevedo JI, Mangone PG. Arthroscopic lateral ankle ligament reconstruction. Tech Foot Ankle Surg 2011;10:111-116.

4. Delfaut EM, Demondion X, Boutry N, et al. Multi-fasciculated anterior talofibular ligament: reassessment of normal findings. Eur Radiol 2003;13(8):1836-1842.

5. Ruth CJ. The surgical treatment of injuries of the fibular collateral ligaments of the ankle. J Bone Joint Surg Am 1961; 43:233-236.

6. Stephens MM, Kelly PM. Fourth toe in flexion sign: a new clinical sign for identification of the superficial nerve. Foot Ankle Int 2000;21(10):860-863.

7. Golanó P, Vega J, Pérez-Carro L, et al. Ankle anatomy for the arthroscopist. Part I: The portals. Foot Ankle Clin N Am 2006; 11(2):253-273

8. Vega J, Marimón J, Golanó P, et al. True submalleolar accessory ossicles causing impingement of the ankle. Knee Surg Sports Traumatol Arthrosc 2010;18(2):254-257.

9. Komenda GA, Ferkel RD. Arthroscopic findings associated with the unstable ankle. Foot Ankle Int 1999;20(11):708-713.

10. Van Dijk CN, Bossuyt PM, Marti RK. Medial ankle pain after lateral ligament rupture. J Bone Joint Surg Br 1996;78(4):562-567.

11. Nery C, Raduan F, Del Buono A, et al. Arthroscopic-assisted Broström-Gould for chronic ankle instability: a long-term follow-up. Am J Sports Med 2011;39(11):2381-2388.

12. Hawkins RB. Arthroscopic stapling repair for chronic lateral instability. Clin Podiatr Med Surg 1987;4(4):875-883.

13. Kashuk KB, Landsman AS, Werd MB, et al. Arthroscopic lateral ankle stabilization. Clin Podiatr Med Surg 1994;11(3): 407-423.

14. Kim ES, Lee KT, Park JS, et al. Arthroscopic anterior talofibular ligament repair for chronic ankle instability with a suture anchor technique. Orthopedics 2011;34(4):1-5. 